\title{
Analysis of Trace Elements in Bone by Radioisotope Induced EDXRF
}

Daisy Joseph, ${ }^{\star}$ Sivan Kutty,† Ajay Chaubey, $¥$ R K Choudhury,§ Astrid Lobo Gajiwala, ${ }^{* *}$ Urmila Samant ${ }^{\dagger \dagger}$ and Cynthia D’Limał

\section{Abstract}

In order to obtain a correlation of lead in bone and osteoporosis and other toxic related disease a study was carried out on bones. Femoral head bone samples obtained from osteoporosis patients were analyzed using $\mathrm{Cd}^{109}$ induced EDXRF consisting of a $\mathrm{Si}(\mathrm{Li}) \mathrm{X}$-ray detector spectrometer coupled to a PC based Multichannel Analyzer. Besides other trace elements, calcium and strontium were predominantly seen in all samples. Lead was also detected.

Keywords: EDXRF, femoral head, bone samples, osteoporosis, lead

\footnotetext{
* Nuclear Physics Division, Bhabha Atomic Research Centre, Trombay, Mumbai-400085; djoseph@barc.gov.in

${ }^{\dagger}$ Medical Division, Bhabha Atomic Research Centre, Trombay, Mumbai400085

‡ Medical Division, Bhabha Atomic Research Centre, Trombay, Mumbai400085

$\S$ Nuclear Physics Division, Bhabha Atomic Research Centre, Trombay, Mumbai-400085

** Tissue Bank, Tata Memorial Hospital, Dr Ernest Borges Road, Mumbai400012

t† Tissue Bank, Tata Memorial Hospital, Dr Ernest Borges Road, Mumbai400012

执 3Tissue Bank, Tata Memorial Hospital, Dr Ernest Borges Road, Mumbai- 400012
} 


\section{Introduction}

Lead is predominantly stored in the human body in calcified tissues [1]. $90-95 \%$ of the total lead burden is contained within bone in non-occupationally exposed adults [2]. The total lead content of bone is reported to be up to $200 \mathrm{mg}$ in 60-70 year old men, less in women [3]. The turnover rate of lead in cortical and trabecular bone is slow. Quantitative estimates of the half-life vary, but there is a consensus that it is of the order of years or even decades. Therefore, through childhood and most of adult life, lead exposure from both environmental and occupational sources results in an increased lead concentration within the bone matrix. A measure of bone-lead content thus reflects integrated or cumulative, and thus long-term or chronic lead exposure and provides a useful indicator of the cumulative dose of lead presented over time to the target organs of lead. In vivo bonelead measurements may therefore clarify the risks associated with lead exposure in two ways. Health effects which are associated with chronic lead exposure may be identified by their correlation with bone-lead level and bone-lead measurements may ultimately allow the identification of subjects at risk from mobilization of their body lead stores and allow appropriate intervention strategies to be devised. Since in vivo bone lead measurements may not be very practical, it is suggested to identify trace element profiles of bone lead by Energy Dispersive X-ray Fluorescence (EDXRF). EDXRF is a analytical technique which can estimate quantitatively the concentrations in any sample (liquid, solid or semi-solid) by obtaining a trace element profile of the sample using a excitation source, X-ray detector coupled to multichannel analyzer (MCA). Lead levels in blood have already been determined by PIXE and it was concluded that a patient with acute anemia has high lead levels in blood leading to low Fe levels [4]. It may be mentioned that Proton Induced X-ray Emission (PIXE) in which protons are used as excitation sources and EDXRF (in which photons from radioisotope sources are used as excitation sources: in the present case $\mathrm{Cd}^{109}$ ) are complimentary techniques. The complimentarity of PIXE and XRF results from the following effects: For a given proton energy the PIXE cross section decreases with increasing $Z$, while the XRF 
cross section increases with $\mathrm{Z}$ until the electron binding energy of the atom reaches the energy of the exciting photons. Similarly the number of photons hitting the target in the case of XRF is about $10^{8} \mathrm{~s}^{-1}$. Whereas in the case of PIXE proton beam having an intensity of $10 \mathrm{nA}, 10^{11}$ to $10^{12} \mathrm{~s}^{-1}$ protons hit the target. In the present investigations, we have used EDXRF as a preliminary study to check the lead levels in bones. In the present study, we have analyzed bone samples of normal patient (patient without osteoporosis) and osteoporosis patients using X-ray spectrometer consisting of a $\mathrm{Cd}^{109}$ radioisotope source, $\mathrm{Si}(\mathrm{Li})$ detector, spectroscopy amplifier and a PC based MCA. The elements $\mathrm{Ca}, \mathrm{Cu}, \mathrm{Br}, \mathrm{Sr}$ and $\mathrm{Zr}$ were detected at ppm levels. Over the years several investigations are made regarding the correlation of bone lead with osteoporosis [5-7]. The following sections will elucidate the methodology and the correlation established for normal and diseased patients.

\section{Materials and methods}

Freeze-dried, irradiated, cortico-cancellous human bone granules were produced from the surgical residues of 10 donors following total hip and total knee replacement surgeries. Donor consent was obtained in all cases. After screening of the donor to eliminate infectious diseases, the bone was cleaned of all soft tissue, cut and washed with saline and then filtered water to remove the blood and bone marrow. This was followed by pasteurization at $60{ }^{\circ} \mathrm{C}$ for 3 hours and soaking in $70 \%$ e thyl a lcohol for 1 hour. The bone was freeze-dried for 48-72 hours to obtain a residual moisture content of less than $8 \%$. The bone powder was then sieved through a standard sieve to give a particle size of approximately 500 microns. The granules were then distributed in vials of 300 milligrams each, using aseptic techniques in a biosafety cabinet. After appropriate labeling the vials were sterilized using a dose of $25 \mathrm{kGy}$ gamma radiation. The powdered bone samples were mixed with cellulose as a binding material to make self-supporting pellets weighing $300 \mathrm{mg}$. A hydraulic press was used to make pellets of $2.5 \mathrm{~cm}$ diameter. The samples in tablet form were then placed in front of a $\mathrm{Si}(\mathrm{Li}) \mathrm{X}$-ray detector spectrometer (Fig. 1). For the excitation of the samples, 
$\mathrm{Cd}^{109}$ radioactive source of strength $20 \mathrm{mCi}$ was employed in annular geometry to prevent the direct exposure of the excitation source to the detector and minimize backscatter interference. $\mathrm{Cd}^{109}$ emits $\mathrm{Ag} \mathrm{K}$ X-rays (energy $22.25 \mathrm{keV}$ ). The X-ray spectra were recorded for a counting time of 2000 seconds and stored in a PC based MCA for further offline analysis.

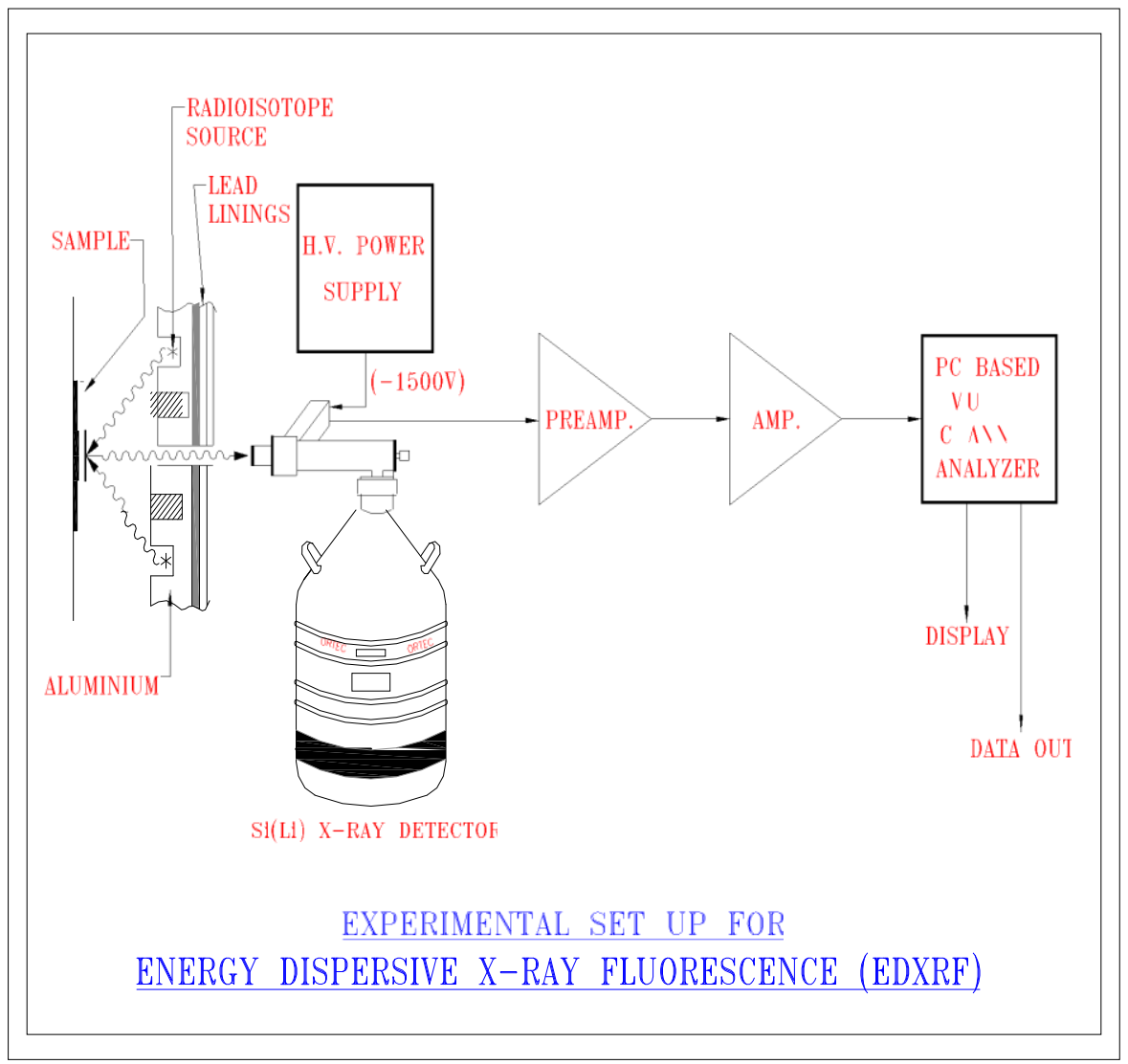

Fig. 1. Experimental set up of EDXRF

The acquired data was Gaussian fitted and the area under the peak was recorded for analysis and quantitative computations. The counts obtained are just the X-ray fluorescent intensity. For it to be converted to concentration values, the following equation should be used. For any element $j$ the intensity of the X-ray line is related to the concentration of the $j_{\text {th }}$ element $\mathrm{m}_{j b y}$ the formula 


$$
I_{j}=I_{0} G k_{j} m_{j} C_{j}
$$

Where $\mathrm{I}_{j}$ the intensity of the $\mathrm{j}^{\mathrm{t}}$ element, $\mathrm{I}_{0}$ is the intensity of the excitation source, $\mathrm{G}$ is the Geometrical factor like the geometry of the sample and the distance of the sample from the source and detector, $k_{j}$ is the excitation cum detection factor. $\mathrm{m}_{j}$ is the concentration of the $\mathrm{j}^{\text {th }}$ element. $\mathrm{C}_{j}$ the absorption factor in the sample. For computation of $I_{0} G$ an external standard $Y$ sample of known concentration of $100 \mathrm{ppm}$ is used in the same geometry as the sample and the Intensity noted. All other factors being the same $I_{0} G$ is calculated.

\section{Results and discussion}

Fig.2(a) is the X-ray spectrum of a bone sample of an osteoporosis patient. Lead L X-rays were detected, while Fig.2(b) is the X-ray spectrum of a bone sample of a normal patient (bone 7). Since this was only a preliminary study, the bone is a case study of a pool sample; hence details could not be produced such as case history of the patient such as age and other diseases. Table 1 gives the concentrations of elements in bone calculated in ppm. Of all the elements detected we see that $\mathrm{Ca}$ is highest followed by $\mathrm{Sr}, \mathrm{Zn}$, $\mathrm{Fe}$ and $\mathrm{Cr}$. $\mathrm{Pb}$ is seen in some patients. It is known that bones and teeth consist of calcium phosphate (apatite) intimately inter grown with an organic matrix, largely composed of protein (collagen and minor amounts of noncollagenous protein). The mineral component has a well defined chemical composition made up of calcium phosphate, hydroxyl and carbonate. Many of the trace elements such as $\mathrm{Sr}, \mathrm{Ba}$ and $\mathrm{Pb}$ are not essential to the diet, but enter the body passively and can be trapped at atomic sites in the bone mineral that are normally occupied by $\mathrm{Ca}$. Sr is a radiologically sought element in bone. As seen from the Table 1, except for one patient all bones have lead in the range of 2-5ppm which is considered high as compared to international standards [8]. $\mathrm{Pb}$ has no biological function and is toxic. Its correlation to the degree of osteoporosis has to be studied clinically. $\mathrm{Zn}$ regulates secretion of calcitonin from thyroid gland and influences on bone turnover. 


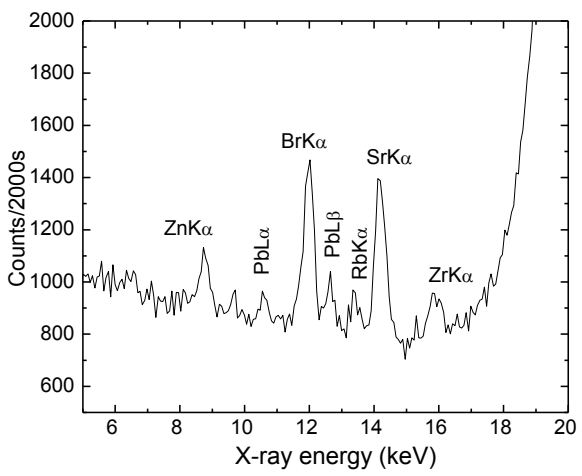

Fig. 2 (a) X-ray spectrum of femoral head of osteoporotic patient from TMH

\begin{tabular}{|l|l|l|l|l|l|l|l|l|l|}
\hline $\begin{array}{c}\text { Trace } \\
\text { elements }\end{array}$ & $\begin{array}{c}\text { Bone } \\
1\end{array}$ & \multicolumn{1}{|c|}{2} & \multicolumn{1}{|c|}{4} & \multicolumn{1}{|c|}{5} & 6 & 7 & 8 & 9 \\
\hline \hline $\mathrm{Ca}$ & $618 \pm 22$ & $817 \pm 11$ & $471 \pm 11$ & $709 \pm 21$ & $1406 \pm 13$ & $491 \pm 12$ & $407 \pm 11$ & $512 \pm 11$ & $453 \pm 12$ \\
\hline \hline $\mathrm{Cr}$ & $3 \pm 0.1$ & $5 \pm 0.2$ & nd & nd & nd & nd & Nd & nd & Nd \\
\hline \hline $\mathrm{Fe}$ & $6 \pm 2$ & $4 \pm 1$ & nd & $3 \pm 0.1$ & $6 \pm 0.2$ & nd & Nd & $2 \pm .2$ & Nd \\
\hline \hline $\mathrm{Zn}$ & $7 \pm 1$ & $6 \pm 1$ & $4 \pm 2$ & nd & $12 \pm 3$ & $3 \pm 0.1$ & $2 \pm 0.1$ & $3 \pm 0.1$ & $3 \pm 0.2$ \\
\hline \hline $\mathrm{Sr}$ & $11 \pm 2$ & $11 \pm 1$ & $7 \pm 1$ & $4 \pm 1$ & $15 \pm 3$ & $6 \pm 1$ & $7 \pm 1$ & $6 \pm 1$ & $11 \pm 1$ \\
\hline \hline $\mathrm{Pb}$ & $5 \pm 1$ & $2 \pm 1$ & $1 \pm 0.1$ & $4 \pm 1$ & $5 \pm 1$ & $2 \pm 1$ & $\mathrm{Nd}$ & $2 \pm 1$ & $1 \pm 1$ \\
\hline
\end{tabular}

Table 1: Concentrations of trace elements in bone samples in ppm

nd - not detected

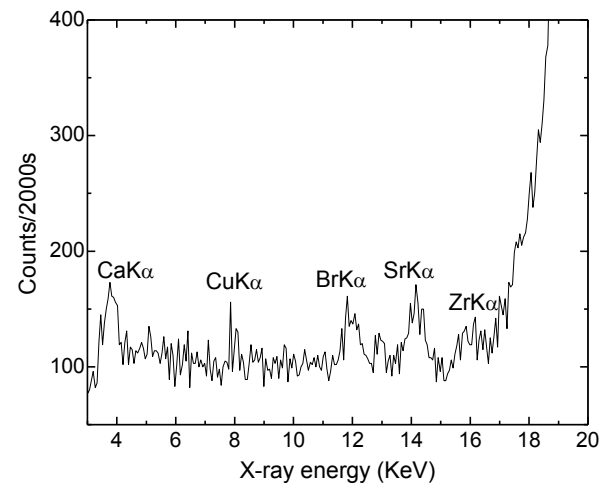

Fig. 2 (b) X-ray spectrum of a normal bone sample 


\section{Conclusion}

Bone samples are easy targets for monitoring long-term exposure of persons to toxic elements such as $\mathrm{Pb} . \mathrm{Pb}$ is a clear indicator of the cause for osteoporosis in patients. It is shown in this work that trace elements in the ppm range can be measured using a rapid, sensitive and non-destructive technique such as radioisotope induced EDXRF.

\section{References}

[1] T. Akyuz, A. Bassari and S. Akyuz, "Determination of elements in bone of tuberculous-arthritis patients by radioisotope X-ray fluorescence analysis," J. Radioanal. Nucl. Chem., vol. 232, p. 253, 1998. http://dx.doi.org/10.1007/BF02383750

[2] A. C. Todd and D. R. Chettle, "In vivo X-ray fluorescence of lead in bone: review and current issues," Environ. Health Perspect., vol. 102, p. 172, 1994.

[3] B. Ytrehus, H. Skagemo, G. Stuve, T. Sivertsen, K. Handeland and T. Vikeren, "Osteoporosis, bone mineralization, and status of selected trace elements in two populations of moose calves in Norway,"Oestoporosis: J. Wildlife Diseases, vol. 35, p. 204, 1999.

[4] M. Lal, D. Joseph, R. K. Choudhury, I. Gauba and H. N. Bajpai, "Studies of blood lead levels in children by proton-induced X-ray emission (PIXE)," Scien. Tot. Environ. vol. 103, p. 209, 1991. http://dx.doi.org/10.1016/0048-9697(91)90146-6

[5] L. Tandon, G. V. Iyengar and R. M. Parr, "A review of radiologically important trace elements in human bones,". Appl. Radiat. Isot., vol. 49, pp. 903, 1998. http://dx.doi.org/10.1016/S0969-8043(97)10102-6

[6] E. K. Silbergeld, J. Schwartz and K. Mahaffey, "Lead and osteoporosis: Mobilization of lead from bone in postmenopausal women," Environ. Res., vol. 47, p. 79, 1988. http://dx.doi.org /10.1016/S0013-9351(88)80023-9

[7] M. H. Dabek, J. Raisanen and J. T. Dabek, "Trace element study of human bone by X-ray emission analysis using an external proton beam", J. Radioanal. Chem., vol. 63, p. 163, 1981. http://dx.doi.org/ 10.1007/BF02518418.

[8] G. V. Iyengar, W. E. Kollmer and H. J. M. Bowen, "The elemental Composition of human Tissues and Body fluids," Verlag Chemie, p. 133, 1978. 